\title{
CARACTERÍSTICAS DO RESÍDUO DO TRITURADOR DE SUCATAS (RTS) DE UMA SIDERÚRGICA BRASILEIRA*
}

\section{Resumo}

\author{
Daniele Perondi ${ }^{1}$ \\ Danielle Restelatto ${ }^{2}$ \\ Christian Manera ${ }^{3}$ \\ Andrezza Piroli Tonello \\ Marcelo Godinho 5 \\ Antônio Cezar Faria Vilela ${ }^{6}$
}

Diferentes resíduos são gerados pelas indústrias siderúrgicas, dentre eles o resíduo dos trituradores de sucatas (RTS). O RTS gerado pelas siderúrgicas brasileiras se difere dos demais países devido aos materiais que são alimentados no triturador. Assim, o objetivo deste trabalho foi avaliar as características do RTS gerado por uma siderúrgica brasileira. As seguintes técnicas foram utilizadas: identificação e quantificação dos principais constituintes, análise termogravimétrica, imediata, elementar, poder calorífico superior e teor de cloro e metais. Os principais polímeros presentes no RTS foram o poliestireno e o polietileno. A partir dos resultados de análise imediata, elementar e de poder calorífico superior do RTS, verificou-se uma elevada quantidade de matéria volátil $(76,22 \% \pm 6,90)$. Os altos teores de carbono $(72,92 \%)$ e hidrogênio $(9,86 \%)$, foram atribuídos à elevada quantidade de polímeros e borrachas presentes no resíduo. O teor de enxofre $(0,21 \%)$ pode ser atribuído a presença de borracha no resíduo, enquanto o teor de cloro $(1,33 \% \pm 0,13)$ pode ser atribuído a presença do PVC no resíduo. O poder calorífico superior do RTS foi de $30,01 \pm 1,22 \mathrm{MJ} / \mathrm{kg}$, de forma que uma avaliação termoquímica mostra-se como uma técnica atrativa para a sua destinação final.

Palavras-chave: Resíduo do triturador de sucata; Siderúrgica brasileira; Técnicas de caracterização.

\section{SHREDDER WASTES CHARACTERISTICS FROM A BRAZILIAN STEEL INDUSTRY}

\section{Abstract}

Different wastes are generated by steel industries, among them the shredder residue (SR). The SR generated by Brazilian steel industries differs from other countries due to the materials that are fed into the shredder. Therefore, the objective of this work was to evaluate the SR characteristics generated by a Brazilian steel industry. The following analyzes were used: identification and quantification of the main constituents, thermal analysis, proximate, ultimate, higher calorific value, chlorine and metals content. The main polymers present in the SR were polystyrene and polyethylene. From proximate, ultimate and higher calorific value analysis results, a high amount of volatile matter $(76.22 \% \pm 6.90)$ was verified. The high levels of carbon $(72.92 \%)$ and hydrogen $(9.86 \%)$ were attributed with the high amount of polymers and rubbers present in the residue. The sulfur content $(0.21 \%)$ can be attributed to the presence of rubber in the residue, while the chlorine content $(1.33 \% \pm 0.13)$ can be attributed to the presence of PVC in the residue. The higher calorific value of SR was $30.01 \pm 1.22 \mathrm{MJ} / \mathrm{kg}$, therefore a thermochemical evaluation is shown as an attractive technique for its final destination.

Keywords: Shedder wastes; Brazilian steel industry; Characterization techniques.

Química, Doutora, Bolsista PDJ/CNPq, Laboratório de Siderurgia/ DEMET/PPGE3M da Escola de Engenharia da Universidade Federal do Rio Grande do Sul, Porto Alegre, Rio Grande do Sul, Brasil. 
2 Estudante de Engenharia Química, Laboratório de Energia e Bioprocessos da Universidade de Caxias do Sul, Caxias do Sul, Rio Grande do Sul, Brasil.

3 Engenheiro Químico, aluno de mestrado do Programa de Pós-Graduação em Engenharia de Processos e Tecnologias da Universidade de Caxias do Sul, Caxias do Sul, Rio Grande do Sul, Brasil.

4 Estudante de Engenharia Química, Laboratório de Energia e Bioprocessos da Universidade de Caxias do Sul, Caxias do Sul, Rio Grande do Sul, Brasil.

5 Engenheiro Químico, Doutor, professor do corpo permanente do programa de Pós-graduação em Engenharia de Processos e Tecnologias, Laboratório de Energia e Bioprocessos da Universidade de Caxias do Sul, Caxias do Sul, Rio Grande do Sul, Brasil.

6 Prof. titular, Dr.-Ing., Laboratório de Siderurgia/DEMET/PPGE3M/ da Escola de Engenharia da Universidade Federal do Rio Grande do Sul, Porto Alegre, Rio Grande do Sul, Brasil. 


\section{INTRODUÇÃO}

No atual estágio de desenvolvimento da sociedade, é impossível imaginar o mundo sem o uso do aço. A produção de aço é um forte indicador do estágio de desenvolvimento econômico de um país. Seu consumo cresce proporcionalmente à construção de edifícios, execução de obras públicas, instalação de meios de comunicação e produção de equipamentos. O aço é hoje o produto mais reciclável e reciclado do mundo. Assim, carros, geladeiras, fogões, latas, barras e arames tornamse sucatas, que alimentam os fornos das usinas, produzindo novamente aço de qualidade [1].

Para que a sucata possa ser utilizada no processo de fabricação do aço, ela precisa ser previamente preparada, de forma que a necessidade de utilização de trituradores de sucatas Shredder tornou-se importante para aumentar a produtividade dos fornos elétricos a arco.

Inicialmente, a sucata é triturada através do triturador (moinho de martelos), e após passa pelos tambores magnéticos, cujo objetivo é a separação da fração ferrosa magnética da não-ferrosa. A fração ferrosa é o produto do triturador. Esse produto é chamado de sucata Shredder ou de sucata triturada e é posteriormente encaminhado para ser carregado em fornos elétricos a arco. A fração não magnética (não-ferrosa) é encaminhada para um processo de separação (Eddy Current: corrente Parasita) que permite a segregação predominantemente do alumínio e do cobre. Tanto o alumínio, quanto o cobre, podem ser comercializados pela própria siderúrgica. A fração não magnética constitui o resíduo do triturador de sucata (RTS). O RTS é direcionado para locais específicos, conforme sua faixa granulométrica (RTS $<15 \mathrm{~mm}$, entre 15 e 100 $\mathrm{mm}$ e $>100 \mathrm{~mm}$ ). A fração do RTS $>100 \mathrm{~mm}$ é o objeto deste trabalho.

O RTS também é denominado de "Auto Shredder Residue (ASR)", "Shredder Fluff (SF) ", "Resíduos de Trituração (RT) ", "Auto fluff" ou simplesmente "fluff" [2]. O RTS é constituído principalmente de polímeros, espumas, vidros, borrachas, têxteis, solo, etc., e não há uma alternativa definitiva para o seu aproveitamento. O destino mais utilizado para o RTS pelas indústrias siderúrgicas brasileiras é o aterro de resíduos industriais, sendo que este tipo de disposição resulta em um alto custo para os geradores. O referido resíduo é classificado segundo a NBR 10.004/2004 [3] como não-inerte (Classe II).

O RTS possui características de uma cadeia de resíduos complexa, o que torna o seu tratamento e reaproveitamento um processo um tanto difícil. Além disto, o RTS gerado pelas siderúrgicas brasileiras se difere dos demais países devido aos materiais que são alimentados no triturador. Comumente, em outros países os trituradores são alimentados com automóveis, ao passo que no Brasil, os materiais alimentados no triturador procedem das mais variadas fontes (produtos da linha branca, como por exemplo geladeiras, fogões, etc.), tornando a composição do resíduo final extremamente heterogênea. Assim, a contribuição científica do presente trabalho está na avaliação das características do resíduo do triturador de sucata de uma siderúrgica brasileira, para fins de um posterior reaproveitamento.

\section{MATERIAIS E MÉTODOS}

As amostras de RTS utilizadas durante o desenvolvimento deste trabalho são provenientes de uma indústria siderúrgica brasileira. Elas foram geradas no triturador de sucata da siderúrgica. O procedimento de coleta foi realizado durante cinco etapas de operação. Os resíduos gerados no processo de trituração da sucata foram 
coletados no início e no final do processo. As amostras foram acondicionadas em sacos (bags) com capacidade de até $600 \mathrm{~kg}$. Tanto o procedimento de amostragem, quanto o procedimento de quarteamento, foram conduzidos de acordo com a NBR 10007/2004 [4].

Após o procedimento de quarteamento, as amostras foram cominuídas em um moinho de facas da empresa Primotécnica, modelo P1001. A redução do tamanho de partícula possibilitou as análises de caracterização. Um fluxograma com as etapas do processo de preparação das amostras é apresentado na Figura 1.

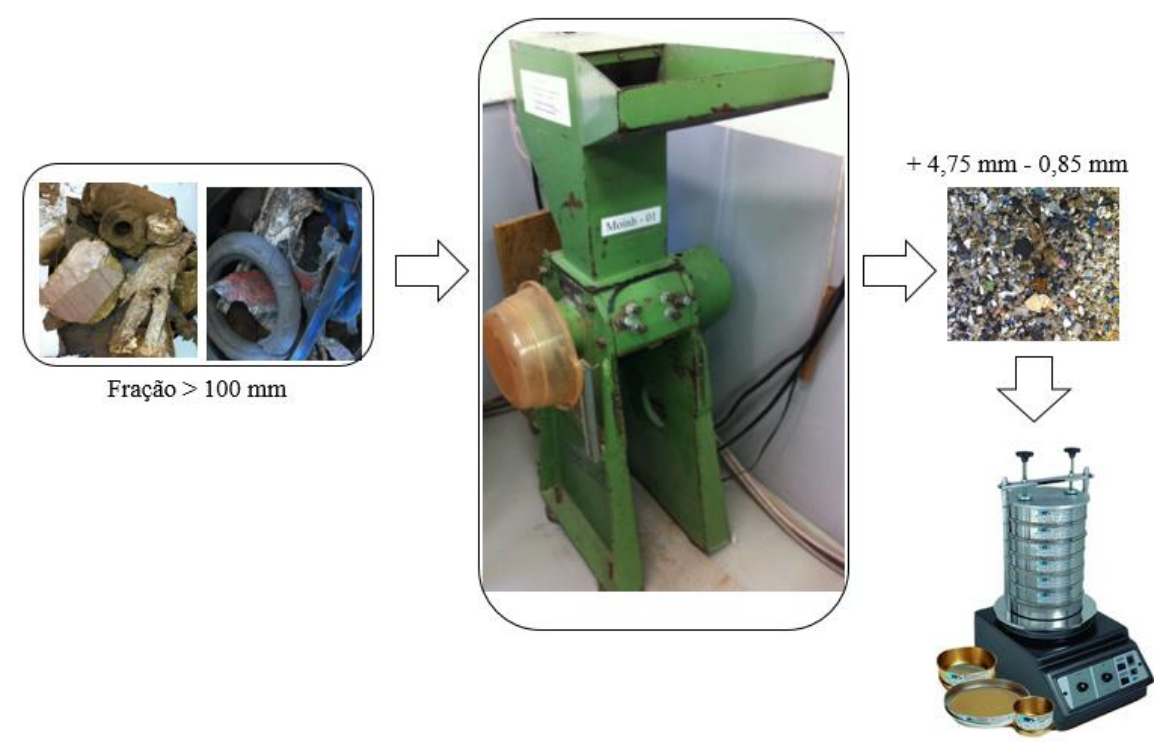

Figura 1. Fluxograma do processo de preparação das amostras.

\subsection{Identificação e quantificação dos materiais presentes no RTS}

Os diferentes materiais presentes no RTS foram inicialmente separados de forma visual, considerando a sua aparência física, conforme pode ser observado na Figura 2. A quantificação foi conduzida em uma amostra de $100 \mathrm{~g}$ do RTS cominuído, e após a separação, as diferentes frações foram pesadas para a determinação da sua participação no RTS.

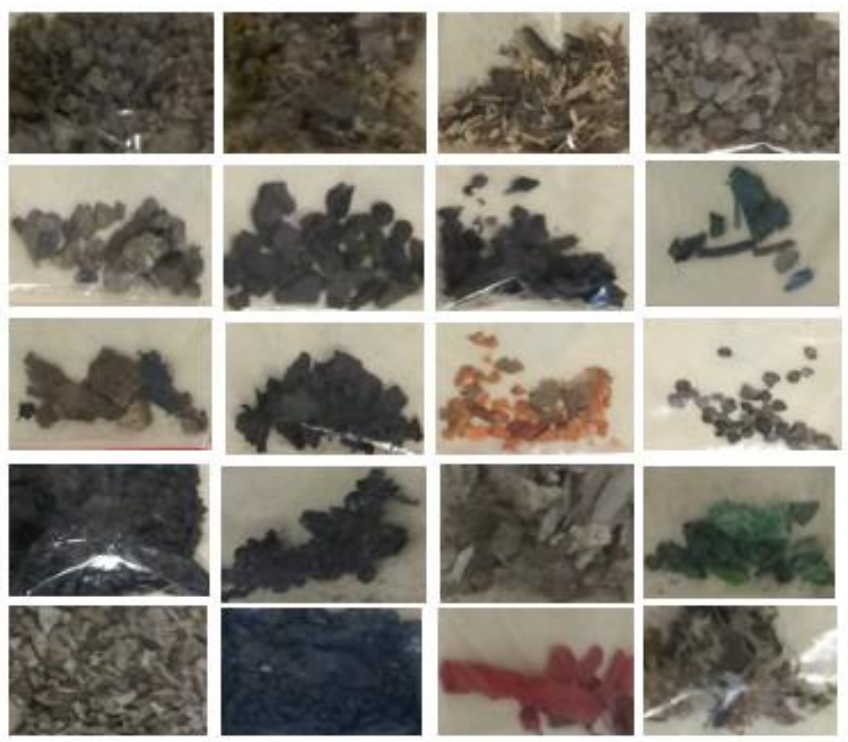

Figura 2. Materiais presentes no RTS. 
Através do teste de coloração da chama e do seu odor foi possível identificar os diferentes polímeros presentes no RTS. De maneira complementar, foi conduzida a análise por Espectroscopia no Infravermelho com Transformada de Fourier (FTIR) para cada um dos polímeros presentes no RTS. A análise de FTIR foi conduzida no equipamento Nicolet IS10 Termo Scientific.

\subsection{Análise termogravimétrica}

As análises térmicas das amostras de RTS, bem como dos principais constituintes do RTS (poliestireno, polietileno de alta densidade, polietileno de baixa densidade, polipropileno, poliamida 6 , poliamida 6.6 e poliamida 6.12), foram realizadas com amostras de diâmetro inferior a $0,9 \mathrm{~mm}$ e massa inicial de aproximadamente $10 \mathrm{mg}$. Os ensaios foram realizados em triplicata, em uma balança termogravimétrica STA 449 F3 Jupiter ${ }^{\circledR}$ da marca Netzsch em atmosfera inerte $\left(\mathrm{N}_{2}\right)$ a uma vazão volumétrica de $50 \mathrm{~mL} / \mathrm{min}$. O cadinho utilizado foi de alumina. Os ensaios não-isotérmicos foram conduzidos com as seguintes taxas de aquecimento: 5 e 35 ${ }^{\circ} \mathrm{C} / \mathrm{min}$ desde a temperatura ambiente até a temperatura final de $800{ }^{\circ} \mathrm{C}$.

\subsection{Análise imediata}

A análise imediata consiste na determinação dos teores de umidade, matéria volátil, cinzas e carbono fixo. As amostras de RTS foram submetidas aos procedimentos descritos nas normas D 3172-89 [5] e D 3173-87 [6] da American Society for Testing and Materials (ASTM).

\subsection{Análise elementar}

A análise elementar das amostras foi realizada no Instituto de Geociências da Universidade Federal do Rio Grande do Sul (UFRGS) segundo as seguintes normas técnicas: ASTM D5373/02 (carbono, hidrogênio e nitrogênio) [7] e ASTM D4239-14e2 (enxofre) [8], utilizando instrumental da ELEMENTAR, modelo Vario Macro.

\subsection{Poder calorífico}

As análises de poder calorífico das amostras de RTS foram conduzidas no Laboratório de Energia e Bioprocessos (LEBIO) da Universidade de Caxias do Sul. O equipamento utilizado foi uma bomba calorimétrica da marca VEB, número 08 , modelo 1031. A norma ASTM D5865 [9] foi adotada.

\subsection{Análise de cloro}

Para a análise de cloro das amostras de RTS, foi utilizada a fração de água deionizada $(5 \mathrm{~mL})$ resultante da combustão realizada para a determinação do poder calorífico das referidas amostras, de acordo com a norma ASTM D4208-02e1 [10] (adaptada). Assim, os $5 \mathrm{~mL}$ de água deionizada resultantes de cada combustão foram utilizados posteriormente para a determinação de cloretos pelo método titulométrico de Mohr.

\subsection{Teor de metais}


As análises do teor de metais presentes no RTS foram conduzidas no Laboratório de Análises e pesquisas Ambientais (LAPAM) da Universidade de Caxias do Sul. A técnica utilizada para a determinação/quantificação foi espectrometria de emissão ótica com plasma acoplado (ICP-OES). Para a realização das análises, fezse necessário o procedimento de preparo por digestão ácida (normas 3050-B [11] e 3120 B [12]).

\section{RESULTADOS E DISCUSSÃO}

\subsection{Identificação e quantificação dos materiais presentes no RTS}

Os resultados da identificação e da quantificação dos materiais presentes no RTS estão apresentados na Tabela 1.

Tabela 1. Identificação e quantificação dos materiais presentes no RTS

\begin{tabular}{|l|r|}
\hline Material & \% (massa) \\
\hline Poliestireno (PS) & 28,69 \\
\hline Polietileno (PE) & 17,85 \\
\hline Finos & 10,00 \\
\hline Metais & 7,83 \\
\hline Poliamida 6 & 5,73 \\
\hline Politereftalato de etileno (PET) & 5,09 \\
\hline Polipropileno (PP) & 4,94 \\
\hline Borracha & 4,90 \\
\hline Madeira & 3,29 \\
\hline Blenda de PP + PE & 2,39 \\
\hline Poliamida 6.12 & 2,21 \\
\hline Espuma vinílica acetinada (EVA) & 2,07 \\
\hline Poliuretano & 1,92 \\
\hline Poliamida 6.6 & 1,77 \\
\hline Poliéster & 0,91 \\
\hline PVC & 0,41 \\
\hline$*$ *btido por diferenca &
\end{tabular}

Através dos resultados apresentados na Tabela 1 é possível verificar que os principais polímeros presentes no RTS são o poliestireno e o polietileno (em torno de $46 \%$ da massa da amostra). A amostra de RTS também possui uma fração significativa de outros polímeros (em torno de $27 \%$ em massa da amostra), além de metais, borracha e madeira.

$\mathrm{Na}$ amostra ainda foi detectada uma fração de partículas de pequenas dimensões, denominadas finos (tamanho de partícula menor do que 2,36 mm) e que não foram possíveis de serem separadas no procedimento de identificação. A referida fração é composta basicamente de terra e areia.

\subsection{Análise termogravimétrica}


A Figura 3 apresenta a curva termogravimétrica (TGA) do RTS e dos principais materiais que constituem o RTS (poliestireno, polietileno de alta densidade, polietileno de baixa densidade, polipropileno, poliamida 6, poliamida 6.6, poliamida 6.12 e madeira) na taxa de aquecimento de $5 \stackrel{\circ}{\circ} \mathrm{C} / \mathrm{min}$.

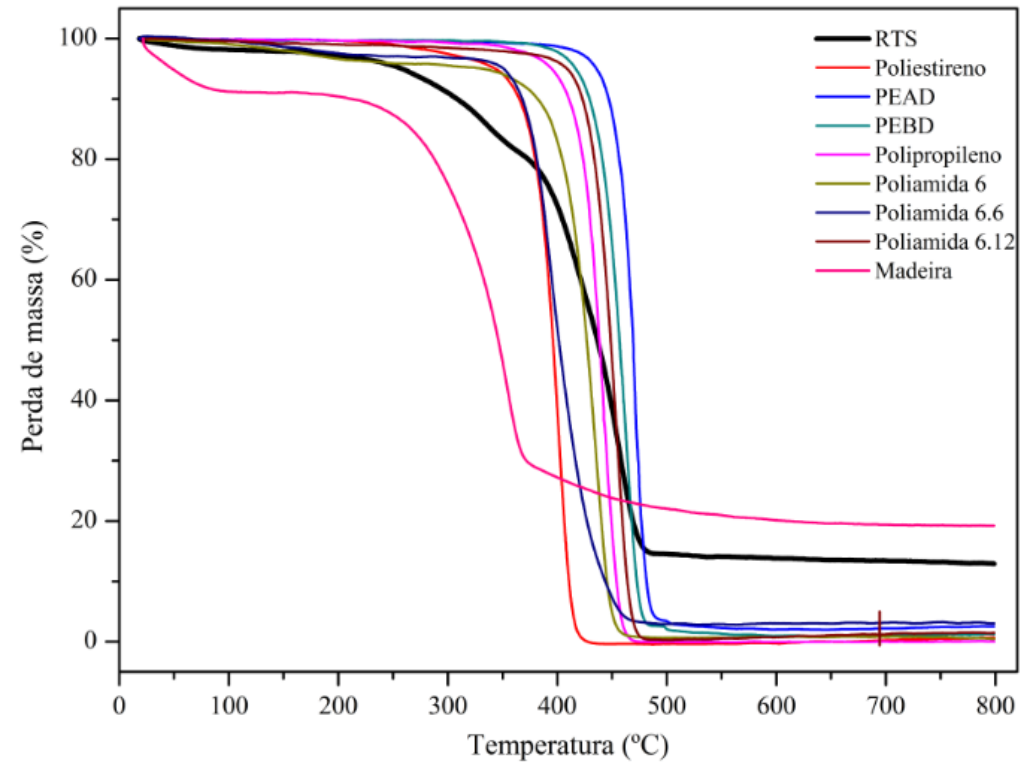

Figura 3. Termograma do RTS e dos materiais constituintes do RTS para a taxa de aquecimento de $5^{\circ} \mathrm{C} / \mathrm{min}$.

Segundo a Figura 3 a amostra de RTS apresenta uma massa residual (massa de amostra remanescente a $800{ }^{\circ} \mathrm{C}$ ) de aproximadamente $17 \%$. Os polímeros presentes na amostra apresentam massa residual insignificante ao final do experimento. Desta forma, a massa residual observada para o RTS deve estar relacionada aos inorgânicos (metais/finos) e ao carbono fixo presentes na madeira, bem como nos demais materiais (como a borracha, por exemplo).

As curvas de derivada primeira (DTG) do RTS nas taxas de 5 e $35^{\circ} \mathrm{C} / \mathrm{min}$, estão apresentadas na Figura 4. Para ambas as taxas de aquecimento, a degradação térmica do RTS ocorre em 3 fases distintas.

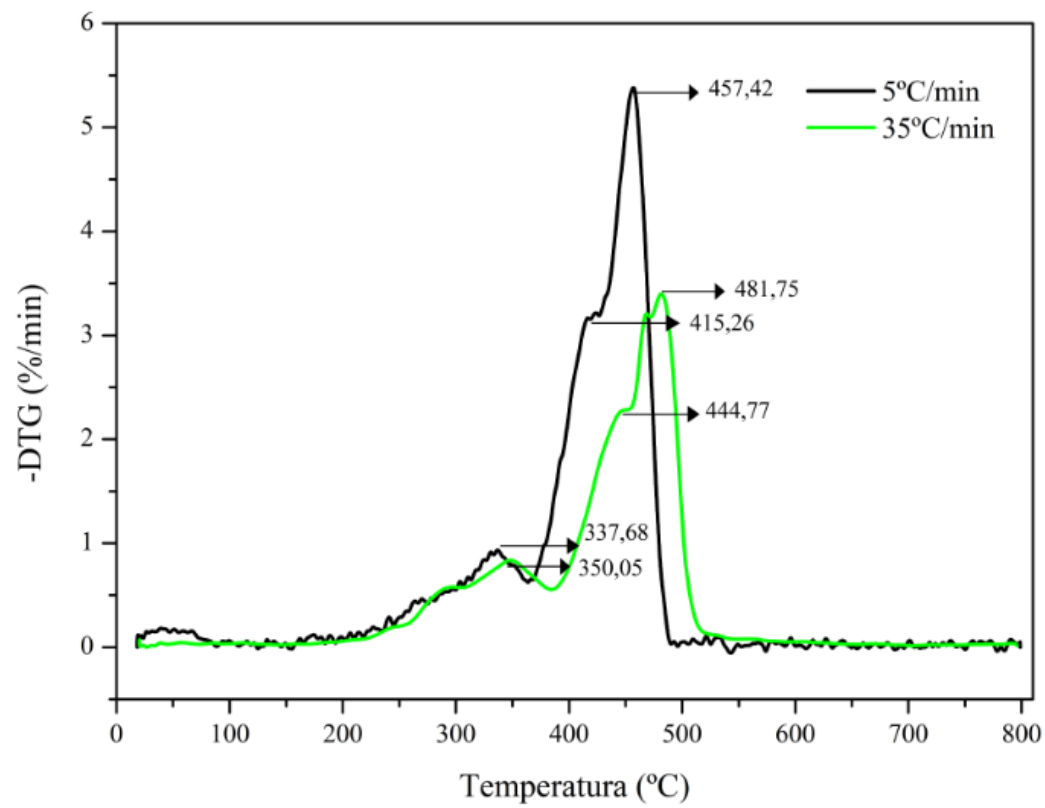

Figura 4. Derivada primeira para a amostra do RTS nas taxas de aquecimento de 5 e $35^{\circ} \mathrm{C} / \mathrm{min}$. 
A primeira fase deve estar associada a degradação térmica da hemicelulose e da celulose presentes na madeira contida no RTS. A formação do ombro na curva DTG a temperaturas mais elevadas indica a degradação do PS contido no RTS e a fase final de degradação térmica está associada a degradação dos demais polímeros presentes no RTS.

\subsection{Análise imediata, elementar e de poder calorífico superior do RTS}

A Tabela 2 apresenta os resultados da análise imediata (matéria volátil, cinzas e carbono fixo), elementar e de poder calorífico superior das amostras de RTS.

Tabela 2. Análise imediata, elementar e de poder calorífico superior do RTS
\begin{tabular}{|l|l|}
\hline Parâmetro & RTS \\
\hline Análise imediata $(\% \mathrm{~m} / \mathrm{m})$ & $76,22 \pm 6,90$ \\
\hline Matéria volátil $(\mathrm{MV})$ & $16,74 \pm 6,86$ \\
\hline Cinzas $(\mathrm{Cz})$ & $7,04 \pm 6,86$ \\
\hline Carbono fixo $(\mathrm{CF})^{* *}$ & 0,21 \\
\hline Análise elementar $\left.{ }^{*} \% \mathrm{~m} / \mathrm{m}\right)$ & 72,92 \\
\hline Enxofre (S) & 9,86 \\
\hline Carbono (C) & 1,45 \\
\hline Hidrogênio $(\mathrm{H})$ & 14,23 \\
\hline Nitrogênio $(\mathrm{N})$ & $1,33 \pm 0,13$ \\
\hline Oxigênio $(\mathrm{O})^{* *}$ & $30,01 \pm 1,22$ \\
\hline Cloro (Cl) & \\
\hline Poder calorífico superior $(\mathrm{MJ} / \mathrm{kg})$ & *base seca
\end{tabular}

**obtido por diferença

Através dos dados apresentados na Tabela 2, é possível verificar que o RTS apresenta elevada quantidade de matéria volátil e baixa quantidade de carbono fixo. As cinzas presentes no RTS são provenientes da matéria inorgânica contida no resíduo, tais como metais, areia e solo. O elevado teor de cinzas encontrado nas amostras de RTS deste trabalho $(16,74 \% \pm 6,86)$, indica que as amostras apresentam uma quantidade importante de inorgânicos.

Os resultados da análise imediata deste trabalho diferem dos obtidos por [13], que obtiveram $54,18 \%$ de matéria volátil (MV) e $36,2 \%$ de cinzas. Esta diferença pode estar associada ao fato dos autores terem caracterizado uma amostra com tamanho de partícula inferior a $2 \mathrm{~mm}$. Outra questão relevante é a composição do resíduo utilizado pelos autores e o resíduo caracterizado neste trabalho. Conforme comentado anteriormente, o resíduo dos trituradores que é produzido no Brasil possui características distintas dos resíduos de trituradores gerados em outros países. Em geral, em outros países os trituradores utilizam somente automóveis em sua alimentação, enquanto os trituradores nacionais são alimentados com diferentes tipos de sucata, incluindo-se as chamadas sucatas de obsolescência, que são muito heterogêneas quanto aos aços e procedências externas as usinas siderúrgicas.

Já os resultados apresentados por [14], que caracterizaram duas amostras provenientes de RTS automotivo apresentam resultados próximos aos encontrados neste trabalho, embora os resíduos tenham diferentes procedências. Os autores encontraram $80,80 \%$ de MV e $19,06 \%$ de cinzas para o resíduo denominado "RTS leve" e, $76,47 \%$ de MV e $22,30 \%$ de cinzas para o resíduo denominado "RTS pesado". 
Com relação a análise elementar, é possível observar que as amostras de RTS apresentam elevados teores de carbono e hidrogênio. Estes resultados estão associados à elevada quantidade de polímeros e borrachas que compõem esta amostra.

A concentração de enxofre $(0,21 \%)$ em sua composição possivelmente está associada a presença de borracha no RTS. Alguns pesquisadores realizaram a análise de enxofre de pneus inservíveis e os teores encontrados por eles variaram de 1,5 a $2,01 \%[15,16]$.

A concentração de cloro no RTS foi de 1,33\%. A presença de cloro é extremamente relevante para processos de tratamento térmico, em especial pela possível formação de dibenzo-para-dioxinas policloradas (PCDD) e dibenzofuranos policlorados (PCDF).

Novamente os resultados obtidos por [13], diferem dos encontrados neste trabalho, principalmente com relação aos teores de carbono e hidrogênio. Os autores encontraram $0,2 \%$ de $\mathrm{S}, 49,5 \%$ de $\mathrm{C}, 5,3 \%$ de $\mathrm{H}$ e $4,5 \%$ de $\mathrm{N}$. Já os resultados obtidos por [17] e [14] para as amostras de RTS pesado, são similares aos obtidos por este trabalho. De Marco et al. [17] encontraram 0,9\% de S, 70,9\% de C, 6,4\% de $\mathrm{H} \mathrm{e} \mathrm{1,2 \%}$ de $\mathrm{N}$, enquanto Joung et al. [14] encontraram $0,14 \%$ de $\mathrm{S}, 64,75 \%$ de $\mathrm{C}, 10,07 \%$ de $\mathrm{H}$ e $0,13 \%$ de $\mathrm{N}$.

O poder calorífico superior do RTS foi de 30,01 $\pm 1,22 \mathrm{MJ} / \mathrm{kg}$. Uma vez que o poder calorífico de um determinado material está relacionado com a quantidade de $\mathrm{C}$ e $\mathrm{H}$ presentes neste material, o valor de poder calorífico superior encontrado, é atribuído ao alto teor de C (72,92\%) e H (9,86\%) do RTS. Os valores de poder calorífico reportados na literatura para o RTS estão compreendidos entre 20 e 30 $\mathrm{MJ} / \mathrm{kg}$ [18].

\subsection{Análise do teor de metais no RTS}

Na Tabela 3 são apresentados os valores médios das concentrações dos metais presentes no RTS.

Tabela 3. Valores médios das concentrações dos metais presentes no RTS

\begin{tabular}{|c|c|c|c|c|c|c|c|c|c|}
\hline \multirow[t]{3}{*}{ Amostra } & \multicolumn{9}{|c|}{ Metais presentes no RTS } \\
\hline & \multicolumn{9}{|c|}{ Resultados expressos em mg/kg } \\
\hline & $\mathrm{Fe}$ & $\mathrm{Zn}$ & $\mathrm{Al}$ & $\mathrm{Cu}$ & $\mathrm{Cr}$ & $\mathrm{Mn}$ & $\mathrm{Pb}$ & $\mathrm{Ni}$ & Co \\
\hline RTS & 22885 & 4827 & 4499 & 2923 & 408 & 362 & 259 & 245 & 5 \\
\hline
\end{tabular}

A presença de metais no RTS apresentou a seguinte ordem de concentração: $\mathrm{Fe} \gg \mathrm{Zn}>\mathrm{Al}>\mathrm{Cu}>\mathrm{Cr}>\mathrm{Mn}>\mathrm{Pb}>\mathrm{Ni}>\mathrm{Co}$. Para fins de comparação, Borjeson et al. [16], encontraram valores próximos para Al (entre 1600 e $8600 \mathrm{mg} / \mathrm{kg}$ ), Cu (entre $1600 \mathrm{e}$ $2800 \mathrm{mg} / \mathrm{kg}$ ) e Zn (entre 1400 e $5300 \mathrm{mg} / \mathrm{kg}$ ). O valor do elemento Pb está abaixo dos reportados por $[19,20]$, assim como do elemento Fe. O Cr apresentou valor próximo ao encontrado por [21] (entre 200 e $400 \mathrm{mg} / \mathrm{kg}$ ) e o Ni apresentou valor próximo ao encontrado por [20] (entre 180 e $930 \mathrm{mg} / \mathrm{kg}$ ).

\section{CONCLUSÕES}

A partir dos resultados de identificação e quantificação, pode-se inferir que os principais polímeros presentes no RTS são o poliestireno e o polietileno (em torno de $46 \%$ da massa da amostra). A amostra de RTS também apresentou uma fração 
significativa de outros polímeros (em torno de $27 \%$ em massa da amostra), além de metais, borracha e madeira. Foi detectada ainda, uma fração de partículas de pequenas dimensões (10\% em massa da amostra), composta basicamente de terra e areia e denominada como finos (tamanho de partícula menor do que 2,36 mm), corroborando com a heterogeneidade da amostra em questão, devido a alimentação inicial do triturador.

A análise termogravimétrica (TGA/DTG) do RTS indicou que a degradação térmica do RTS ocorre em 3 fases distintas. A primeira fase está associada a degradação térmica da hemicelulose e da celulose presentes na madeira contida no RTS, enquanto a segunda fase está associada a degradação térmica do poliestireno, e a última fase a degradação térmica dos demais polímeros presentes no RTS.

A partir dos resultados de análise imediata, elementar e de poder calorífico do RTS, verificou-se uma elevada quantidade de matéria volátil $(76,22 \% \pm 6,90)$ e uma baixa quantidade de carbono fixo $(7,04 \% \pm 6,86)$. O RTS apresentou elevados teores de carbono $(72,92 \%)$ e hidrogênio $(9,86 \%)$, possivelmente associados à elevada quantidade de polímeros e borrachas presentes no resíduo. O enxofre $(0,21 \%)$ presente no RTS pode ser atribuído a presença de borracha no resíduo, enquanto o cloro $(1,33 \% \pm 0,13)$ pode ser atribuído a presença do PVC no resíduo. O poder calorífico superior do RTS foi de $30,01 \pm 1,22 \mathrm{MJ} / \mathrm{kg}$, de forma que uma avaliação termoquímica mostra-se como uma técnica atrativa para a sua destinação final.

\section{Agradecimentos}

Ao Conselho Nacional de Desenvolvimento Científico e Tecnológico por ter financiado e possibilitado esta pesquisa, através do Processo CNPq nํ142141/20135 e a empresa Gerdau Aços Longos S.A, por ter disponibilizado as amostras de RTS.

\section{REFERÊNCIAS}

1 Instituto Aço Brasil. 2016 [acesso em 31 out.2016]. Disponível em: http://www.acobrasil.org.br.

2 Reckziegel VN. Caracterização para o aproveitamento do resíduo de um triturador de sucata em uma usina siderúrgica. Dissertação de mestrado do Programa de PósGraduação em Engenharia de Minas, Metalurgia e de Materiais da Universidade Federal do Rio Grande do Sul, 2012.

3 ABNT. Associação Brasileira de Normas Técnicas. Resíduos Sólidos- Classificação. NBR 10.004, 2004.

4 ABNT. Associação Brasileira de Normas Técnicas - Amostragem de Resíduos Sólidos. NBR 10.007, 2004.

5 Annual Book of ASTM Standards, Vol. 05.06, Standard Practice of Proximate Analysis of Coal and Coke, American Society of Testing Materials, West Conshohocken, PA. D317289, 1997.

6 Annual Book of ASTM Standards, Vol. 05.06, Standard Test Method for Moisture in the Analysis Sample of Coal and Coke, American Society of Testing Materials, West Conshohocken, PA. D3173-87, 1996.

7 Annual Book of ASTM Standards, Vol. 05.06, Standard Test Method for Instrumental Determination of Carbon, Hydrogen and Nitrogen in Laboratory Samples of Coal and Coke, American Society of Testing Materials, West Conshohocken, PA, 2002. D5373-02.

8 Annual Book of ASTM Standards, Vol. 05.06, Standard Test Method for Sulfur in the Analysis Sample os Coal and Coke Using High-Temperature Tube Furnace Combustion, West Conshohocken, PA, 2014. D4239-14e2. 
9 Annual Book of ASTM Standards, Vol. 05.06, Standard Method for Gross Calorific Value of Coal and Coke, American Society of Testing Materials, West Conshohocken, PA. D5865, 2013.

10 Annual Book of ASTM Standards, Vol. 05.06, Standard Test Method for Total Chlorine in Coal by Oxygen Bomb Combustion/Ion Selective Electrode Method, West Conshohocken, PA, 2013. D4208-02e1.

11 US Environmental Protection Agency (EPA), 3050-B Method, revision 02, Acid digestion of sediments, sludges, and soils, 1996.

12 Standard Methods for Examination of Water and Wastewater, 3120 B Method, Metals by plasma emission Spectroscopy, 22 ND Edition, 2012.

13 Mirabile D, Pistelli MI, Marchesini M, Falciani R, Chiapelli L. Thermal valorization of automobile shredder residue: injection in blast furnace. Waste Management. 2002; 22 (8): 841-851.

14 Joung HT, Seo YC, Kim KH. Distribution of dioxins, furans, and dioxin-like PCBs in solid products generated by pyrolysis and melting of automobile shredder residues. Chemosphere. 2007; 68: 1636-1641.

15 Islam MR, Joardder MUH, Hasan SM, Takai K, Haniu H. Feasibility study for thermal treatment of solid tire wastes in Bangladesh by using pyrolysis technology. Waste Management. 2011; 31: 2142-2149.

16 Fernández AM, Barriocanal C, Alvarez RAM. Pyrolysis of a waste from the grinding of scrap tyres. Journal of Hazardous Materials. 2012; 203- 204: 236- 243.

17 De Marco I, Caballero BM, Cabrero MA, Laregoiti MF, Torres A, Chomón MJ. Recycling of automobile shredder residues by means of pyrolysis. Journal of Analytical and Applied Pyrolysis. 2007; 79: 403-408.

18 Harder MK, Forton OT. A critical review of developments in the pyrolysis of automotive shredder residue. Journal of Analytical and Applied Pyrolysis. 2007; 79: 387-394.

19 Borjeson L, Lofvenius G, Hjelt M, Johansson S, Marklund S. Characterization of automotive shredder residues from two shredding facilities with different refining processes in Sweden. Waste Management \& Research. 2000; 18: 358-366.

20 Roy C, Chaala A. Vacuum pyrolysis of automobile shredder residues. Resources, Conservation and Recycling. 2001; 32: 1-27.

21 Day M, Graham J, lachmansingh R, Chen E. Characterization of auto shredder residue - a Canadian perspective1. Resources Conservation and Recycling. 1993; 9 (4): 255279. 